\title{
Editorial
}

\section{Quality Control on Herbal Medicine and Its Application}

\author{
Gallant Kar-Lun Chan ${ }^{(D)},{ }^{1}$ Rentian Wu, ${ }^{2}$ Vicky Ping Chen, ${ }^{2}$ \\ Kevin Yue $\mathrm{Zhu},{ }^{3}$ and Yingqing $\mathrm{Du}^{4}$ \\ ${ }^{1}$ Division of Life Science and Center for Chinese Medicine, The Hong Kong University of Science and Technology, \\ Clear Water Bay Road, Clear Water Bay, Hong Kong \\ ${ }^{2}$ Department of Molecular Pharmacology and Experimental Therapeutics, Mayo Clinic, Rochester, MN 55905, USA \\ ${ }^{3}$ Jiangsu Key Laboratory for High Technology Research of TCM Formulae and Jiangsu Collaborative Innovation Center of Chinese \\ Medicinal Resources Industrialization, State Key Laboratory Cultivation Base for TCM Quality and Efficacy, \\ Nanjing University of Chinese Medicine, Nanjing, Jiangsu, China \\ ${ }^{4}$ Hanshan Normal University, Chaozhou, Guangdong 521041, China
}

Correspondence should be addressed to Gallant Kar-Lun Chan; gallant@ust.hk

Received 28 January 2018; Accepted 29 January 2018; Published 15 March 2018

Copyright (C) 2018 Gallant Kar-Lun Chan et al. This is an open access article distributed under the Creative Commons Attribution License, which permits unrestricted use, distribution, and reproduction in any medium, provided the original work is properly cited.

In this issue, you will see how researchers applied systematic biological method to illustrate the importance of genuineness, so-called "Daodi," of herbal medicinal material. Metabolomics study on herbal medicine as well as their interactions with commonly used compound medicine, such as Aspirin, will also be demonstrated. Curcumin was known to be a chemical compound with many strong biological functions. We will show you an advanced extraction methods which helps establishing quality control standards for herbal medicine with Curcumin. Moreover, the detailed descriptions of clinical effect and safety of herbal medicinal formulation will be covered in this issue. Last but not least, a fast and innovative drug screening platform, namely HerboChips, will be introduced. The paper describes how HerboChips makes drug screening down to molecular level from herbal medicine become feasible.

Although the background of the authors varies, they share one goal-to unmask the beauty of herbal medicine, a system which support our health and population for more than several thousands of years in human history.

\section{Acknowledgments}

Here, we would like to thank the editorial board of eCAM who provided us with the chance to manage this special issue on promoting herbal medicine. The professional support team and user-friendly system make our editorial works with ease. Special thanks should be given to all authors who contributed their papers including those that have not been published in this special issue. The acceptance rate of this special issue ( 6 out of 20) matches the journal acceptance rate (i.e., 29\%), implying that all the submitted works are in high quality. We wish you all enjoy this quality controlled special issue.
Gallant Kar-Lun Chan Rentian $W u$ Vicky Ping Chen Kevin Yue Zhu Yingqing Du




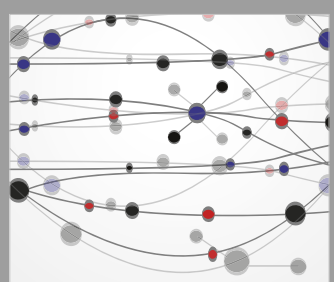

The Scientific World Journal
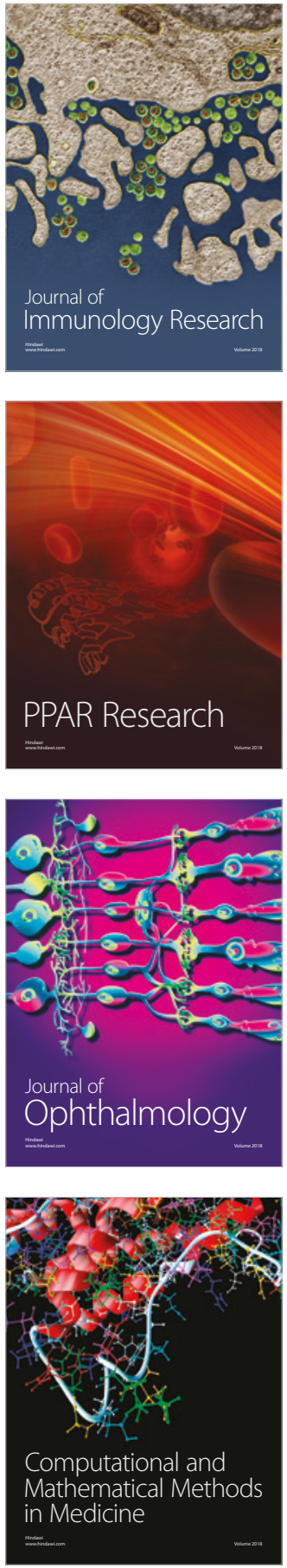

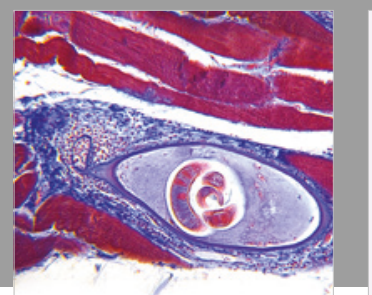

Gastroenterology Research and Practice

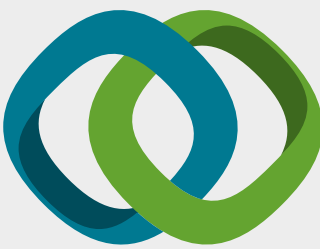

\section{Hindawi}

Submit your manuscripts at

www.hindawi.com
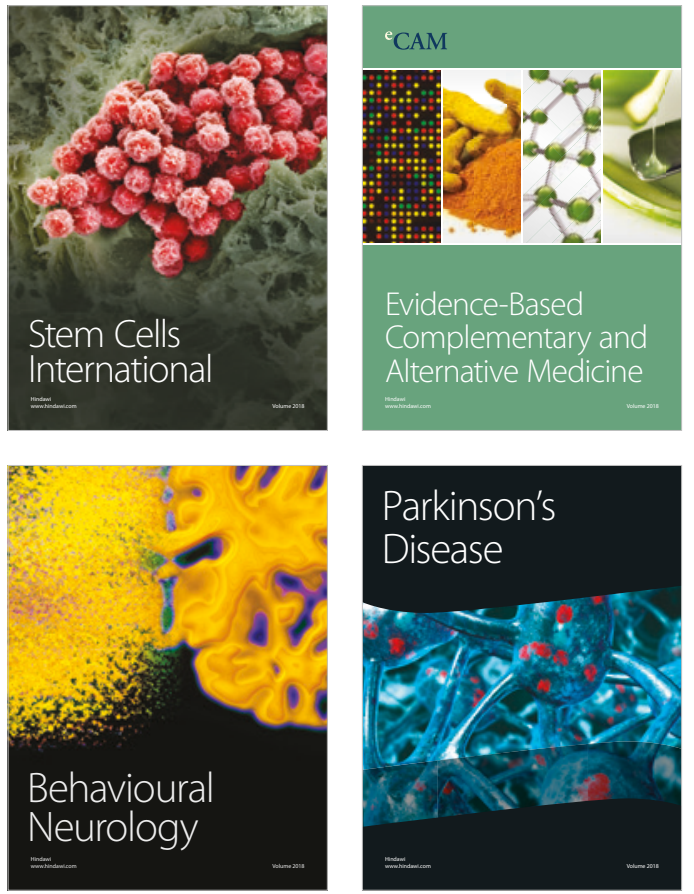

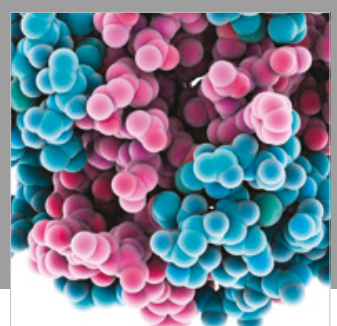

ournal of

Diabetes Research

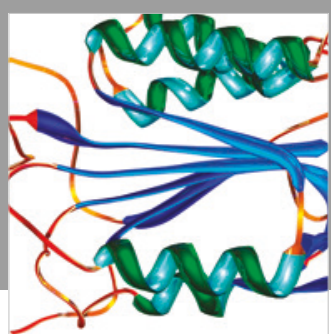

Disease Markers
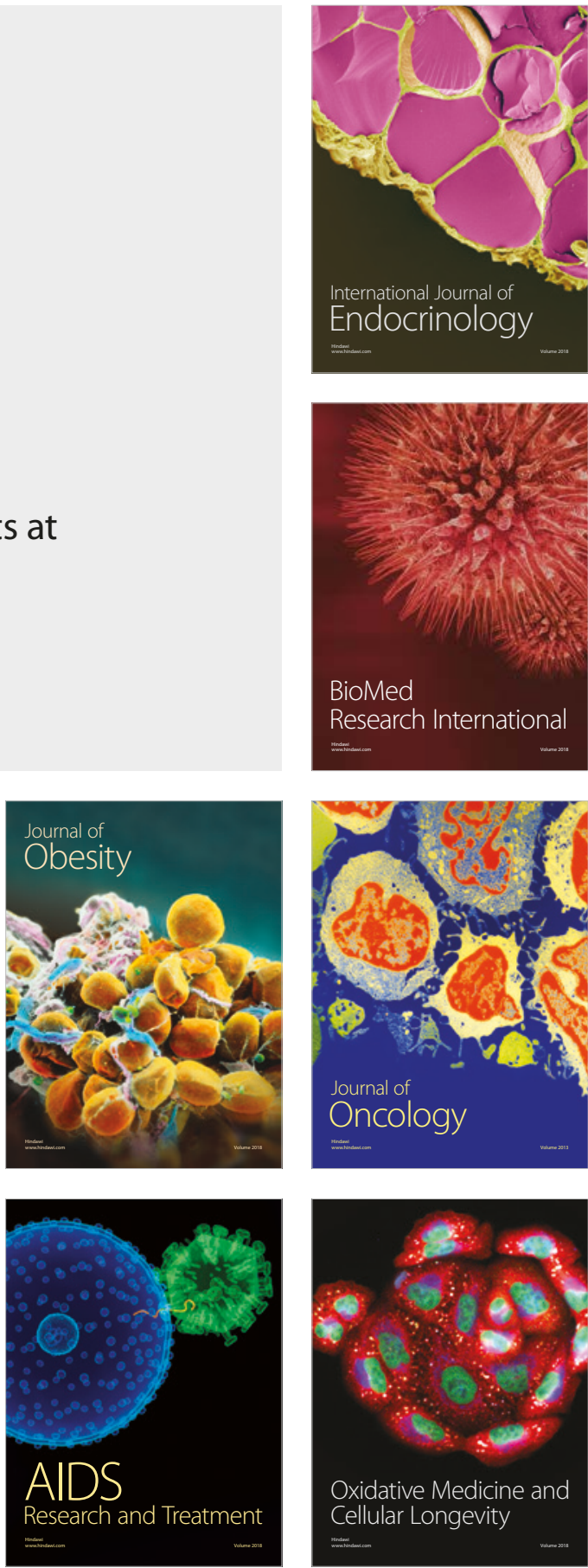